\title{
Research Article \\ Total Stability Properties Based on Fixed Point Theory for a Class of Hybrid Dynamic Systems
}

\author{
M. De la Sen \\ Department of Electricity and Electronics, Institute of Research and Development of Processes, \\ Faculty of Science and Technology, University of the Basque Country, Campus de Leioa (Bizkaia), \\ 644 Apertando de Bilbao, 48080 Bilbao, Spain \\ Correspondence should be addressed to M. De la Sen, manuel.delasen@ehu.es
}

Received 26 March 2009; Accepted 20 May 2009

Recommended by Juan J. Nieto

Robust stability results for nominally linear hybrid systems are obtained from total stability theorems for purely continuous-time and discrete-time systems by using the powerful tool of fixed point theory. The class of hybrid systems dealt consists, in general, of coupled continuous-time and digital systems subject to state perturbations whose nominal (i.e., unperturbed) parts are linear and, in general, time-varying. The obtained sufficient conditions on robust stability under a wide class of harmless perturbations are dependent on the values of the parameters defining the overbounding functions of those perturbations. The weakness of the coupling dynamics in terms of norm among the analog and digital substates of the whole dynamic system guarantees the total stability provided that the corresponding uncoupled nominal subsystems are both exponentially stable. Fixed point stability theory is used for the proofs of stability. A generalization of that result is given for the case that sampling is not uniform. The boundedness of the state-trajectory solution at sampling instants guarantees the global boundedness of the solutions for all time. The existence of a fixed point for the sampled state-trajectory solution at sampling instants guarantees the existence of a fixed point of an extended auxiliary discrete system and the existence of a global asymptotic attractor of the solutions which is either a fixed point or a limit $n$ globally stable asymptotic oscillation.

Copyright (C) 2009 M. De la Sen. This is an open access article distributed under the Creative Commons Attribution License, which permits unrestricted use, distribution, and reproduction in any medium, provided the original work is properly cited.

\section{Introduction}

Stability of both continuous-time and discrete-time singularly perturbed dynamic systems has received much attention [1-5]. Also, stability analysis of discrete-time singularly perturbed systems with calculations of parameter bounds has been reported in $[2,3]$. An assumption used in previous work to carry out the stability analysis of singularly perturbed systems is relaxed in [1] where an upper-bound on the singular perturbation parameters is included to derive such an analysis. On the other hand, the so-called hybrid models 
are a very important tool for analysis in the modern computers and control technologies since they describe usual situations where continuous-time and either discrete-time and/or digital systems are coupled [6,7]. A usual example, very common in practice, is the case when a digital controller operates over a continuous-time plant to stabilize it or to improve its performance. The systems described in [6,7] have a more general structure since the controlled plant can also possess an hybrid nature since all the continuous-time and digital state-variables can be mutually coupled and to possess internal delays [8-10]. An important class of hybrid systems of wide presence in technological applications like telecommunication, teleoperation, or control of continuous systems by discrete controllers to which the above structure belongs to is that consisting of coupled continuous-time and digital or discrete-time subsystems.

On the other hand, fixed point theory and related techniques are also of increasing interest for solving a wide class of mathematical problems where convergence of a trajectory or sequence to some set is essential (see, e.g., [11-16]). Some of the specific topics covered are, as follows.

(1) The properties of the so-called $n$ time reasonably expansive mapping are investigated in [11] in complete metric spaces $(X, d)$ as those fulfilling the property that $d\left(x, T^{n} x\right) \geq h d(x, T x)$ for some real constant $h>1$. The conditions for the existence of fixed points in such mappings are investigated.

(2) Strong convergence of the well-known Halpern's iteration and variants is investigated in [12] and some of the references there in.

(3) Fixed point techniques have been recently used in [14] for the investigation of global stability of a wide class of time-delay dynamic systems which are modeled by functional equations.

(4) Generalized contractive mappings have been investigated in [15] and references there in, weakly contractive and nonexpansive mappings are investigated in [16] and references there in.

(5) The existence of fixed points of Liptchitzian semigroups has been investigated, for instance, in [13].

In this paper, stability results are obtained for a wide class of hybrid dynamic systems whose nominal (i.e., unperturbed) parts are linear and, in general, time-varying while the state perturbations are allowed to be, in general, nonlinear, time-varying, and of a dynamic nature. Those dynamic systems consist of two coupled parts, one being of a continuoustime nature being modeled by an ordinary differential equation and the other one being of a digital nature and is modeled by a difference equation. Both equations are mutually coupled and, respectively, described alternatively by a set of first-order continuous-time differential equations equal to the order of the continuous-time substate and a set of first-order difference equations equal to the order of the digital substate. The results about robust stability (i.e., stability under tolerance to a certain amount of perturbations) are obtained by first obtaining sufficient type stability conditions related to total stability for an extended discrete system which describes the overall state trajectory at sampling instants via the discretization of the continuous-time substate (i.e., the state variables which describe the continuous-time component). Subsequently, a result about total stability of the continuous-time substate is carried out to ensure the system's stability during the intersample intervals. Some links with the results given in [1] about singularly perturbed systems are also given for a special hybrid system within the given class. Finally, some of the results are extended for the case when the 
sequence of sampling periods is allowed to be time-varying. The main technique employed for deriving the stability results is based on the use of fixed point theory.

Notation 1 . $\mathbb{Z}$ and $\mathbb{R}$ are the sets of integer and real numbers, $\mathbb{Z}_{+}:=\{z \in \mathbb{Z}: z>0\}, \mathbb{Z}_{0_{+}}:=$ $\{z \in \mathbb{Z}: z \geq 0\}, \mathbb{R}_{+}:=\{r \in \mathbb{R}: r>0\}, \mathbb{R}_{0+}:=\{r \in \mathbb{R}: r \geq 0\}$.

Also, $\lambda_{\max }(M)$ and $\operatorname{det}(M)$ denote, respectively, the maximum eigenvalue and determinant of the square matrix $M=\left(M^{(i j)}\right)$. The symbol $\otimes$ denotes the direct Kronecker product of matrices. Particular norms for functions, sequences, or matrices are denoted by the appropriate subscript. In the expressions being valid for any norms, those subscripts are omitted.

\section{Problem Statement}

\subsection{Hybrid Dynamic System $\Sigma$}

Consider the following, in general, time-varying dynamic hybrid system.

\section{System $\Sigma$.}

$$
\begin{aligned}
\dot{x}_{c}(t) & =A_{c}(t) x_{c}(t)+A_{c d}(t) x_{d}[k]+\delta_{c}(t), \\
x_{d}[k+1] & =A_{d}[k] x_{d}[k]+A_{d c}[k] x_{c}[k]+\delta_{d}[k], \\
\delta_{c}(t) & =f_{c c}\left(t, x_{c}(t)\right)+f_{c d}\left(t, x_{d}[k]\right)+g_{c c}\left(t, x_{c}(t)\right)+g_{c d}\left(t, x_{d}[k]\right), \\
\delta_{d}[k] & =f_{d c}\left(k, x_{c}[k]\right)+f_{d d}\left(k, x_{d}[k]\right)+g_{d c}\left(k, x_{c}[k]\right)+g_{d d}\left(k, x_{d}[k]\right),
\end{aligned}
$$

for all time $t \in[k T,(k+1) T)$ and discrete time integer index $k \geq 0$ for sampling period $T$ where $x_{c}(t)$ and $x_{d}[k]$ are, respectively, the $n_{c}$ continuous-time (or analog) substate and $n_{d}$ discrete-time (or digital) substate. The continuous-time and discrete-time variables are denoted by $(t)$ and $[k]$, respectively. The discretized analog substate at sampling instants is denoted as a digital signal, that is, $x_{c}(k T)=x_{c}[k]$. The matrix functions $A_{c}(t), A_{c d}(t), A_{d}[k]$, and $A_{d c}[k]$ are of orders being compatible with the corresponding vectors in (1)-(2). Also, $\delta_{c}(t)$ and $\delta_{d}[k]$ are disturbances being, in general, nonlinear and time-varying subject to the following set of constraints on the real vector functions $f_{(\cdot)}$ and $g_{(\cdot)}$.

\section{Constraints $C$.}

(C1) It holds that $A_{d}[k], A_{d c}[k], f_{d c}\left(k, x_{c}[k]\right), f_{d d}\left(k, x_{d}[k]\right), g_{d c}\left(k, x_{c}(t)\right), g_{d d}\left(k, x_{d}[k]\right)$ denote matrix and vector sequences of $k$ of bounded entries. The entries of $A_{c}(t), A_{c d}(t), f_{c c}\left(t, x_{c}(t)\right), f_{c d}\left(t, x_{d}[k]\right), g_{c c}\left(t, x_{c}(t)\right)$, and $g_{c d}\left(t, x_{d}[k]\right)$ are locally integrable functions of $t$ for each fixed $x$ in the closed ball centred at zero $\bar{B}(0, r):=$ $\left\{x \in \mathbb{R}^{n}:\|x\| \leq r\right\} \subset \mathbb{R}^{n}, \operatorname{Max}\left(\left\|x_{c}\right\|,\left\|x_{d}\right\| \leq r\right)$ and all integer $k \geq 0$ and all $t \geq 0$.

(C2) Also, The following hold:

$$
f_{c c}(t, 0)=f_{d c}(t, 0)=0 \in \mathbb{R}^{n_{c}} ; \quad f_{d c}(k, 0)=f_{d d}(k, 0)=0 \in \mathbb{R}^{n_{d}}
$$


(C3)

$$
\begin{aligned}
\left\|f_{c c}\left(t, x_{c 1}\right)-f_{c c}\left(t, x_{c 2}\right)\right\| & \leq \beta_{c c}^{f}\left\|x_{c 1}-x_{c 2}\right\| ; \\
\left\|f_{c d}\left(t, x_{c 1}\right)-f_{c d}\left(t, x_{c 2}\right)\right\| & \leq \beta_{c d}^{f}\left\|x_{c 1}-x_{c 2}\right\|, \\
\left\|f_{d c}\left(k, x_{d 1}\right)-f_{d c}\left(k, x_{d 2}\right)\right\| & \leq \beta_{d c}^{f}\left\|x_{d 1}-x_{d 2}\right\| ; \\
\left\|f_{d d}\left(k, x_{d 1}\right)-f_{d d}\left(k, x_{d 2}\right)\right\| & \leq \beta_{d d}^{f}\left\|x_{d 1}-x_{d 2}\right\| ;
\end{aligned}
$$

$(\mathrm{C} 4)$

$$
\begin{aligned}
\left\|g_{c c}\left(t, x_{c 1}\right)\right\| & \leq \beta_{c c}^{g} r ; \\
\left\|g_{c d}\left(t, x_{d 1}\right)\right\| & \leq \beta_{c d}^{g} r ; \\
\left\|g_{d c}\left(t, x_{c i}\right)\right\| & \leq \beta_{d c}^{g} r ; \\
\left\|g_{d d}\left(t, x_{d i}\right)\right\| & \leq \beta_{d d}^{g} r ;
\end{aligned}
$$

for all $\left\|x_{c i}\right\| \leq r,\left\|x_{d i}\right\| \leq r$ and all integer $k \geq 0$ and all $t \geq 0$, with $h$ being any of the vector real functions or sequences of (2.3) and (2.4) and $\beta_{(\cdot)}^{h}(h=f$ or $h=g$ ) being known nonnegative real constants. It turns out from Picard-Lindeloff theorem that the hybrid dynamic system (2.1)-(2.5) has a unique solution under the set of constraints $C$. The solution is almost everywhere continuous and differentiable but it has bounded discontinuities in general at the sampling instants $t_{k}=k T$; for all $k \in \mathbb{Z}_{0+}$. The problem dealt with in this brief is the investigation of the robust stability of $\Sigma$ (i.e., that of (2.1) and (2.2) with dynamic state disturbances (2.3) and (2.4)) subject to the set of constraints $C$. For this purpose, the state-trajectory of $\Sigma$ at sampling instants is calculated in the following subsection.

\subsection{Extended Discrete System $\Sigma_{d}$}

Direct calculation of the solution of $\Sigma$ at sampling instants (i.e., $t=k T$; for all $k \in \mathbb{Z}_{0_{+}}$) yields the following discrete extended system:

$$
\Sigma_{d}: x[k+1]=A[k] x[k]+\delta[k], \quad \text { all integer } k \geq 0,
$$

with $x[k]=\left(x_{c}^{T}[k], x_{d}^{T}[k]\right)^{T}$ subject to $x[0]=\left(x_{c}^{T}[0], x_{d}^{T}[0]\right)^{T}$, with $x_{c}[0]=x_{c}(0)$, and

$$
\begin{aligned}
& A[k]=\left[\begin{array}{cc}
\Phi_{c}[k] & \Gamma_{c}[k] \\
A_{d c}[k] & A_{d}[k]
\end{array}\right] ; \quad \Gamma_{c}[k]=\int_{0}^{T} \Phi_{c}((k+1) T, k T+\tau) A_{c d}(k T+\tau) d \tau, \\
& \delta[k]=\left(\delta_{c}^{\prime T}[k], \delta_{d}^{T}[k]\right)^{T}=\left(\int_{0}^{T} \delta_{c}^{T}(k T+\tau) \Phi_{c}^{T}((k+1) T, k T+\tau) d \tau, \delta_{d}^{T}[k]\right)^{T},
\end{aligned}
$$


$\Phi_{c}(t)$ and $\Gamma_{c}(t)$ being defined at sampling instants as $\Phi_{c}[k]=\Phi_{c}(k T)=\Psi_{c}((k+1) T, k T)$ and $\Gamma_{c}[k]=\Gamma_{c}(k T)$ are the $k$ th intersample state transition and control matrices of the continuous subsystem, respectively, (i.e., $\dot{\Psi}_{c}(t, 0)=A_{c}(t) \Psi_{c}(t, 0) ; \Psi_{c}(0,0)=I_{n_{c}}$ for all $t \in[k T,(k+1) T)$ and all integer $k \geq 0$.

\section{Main Rsults}

The robust stability of $\Sigma$ subject to the constraints $C$ under the knowledge of the constants $\beta_{(\cdot)}^{(\cdot)}$ is now investigated. The results on robust stability are useful for both local and global stability in the sense that stability is ensured for initial conditions of (2.1)-(2.4) being constrained to the balls $\left\|x_{c}(0)\right\| \leq r,\left\|x_{d}[0]\right\| \leq r$ where the radius $r$ is arbitrary but compatible with the validity of the constraints $C$ on $\Sigma$.

\subsection{Exponential Stability of the Nominal Extended System $\Sigma_{d}^{*}$}

The nominal $\Sigma$ is defined by zeroing $\delta_{c}(t)$ and $\delta_{d}[k]$ in (1)-(2). This results into the nominal version $\Sigma_{d}^{*}$ of $\Sigma_{d}$ in (2.10)-(2.12) satisfying $x^{*}[k+1]=A[k] x^{*}[k]$ with $x^{*}[0]=\left(x_{c}^{T}[0], x_{d}^{T}[0]\right)^{T}$. The following assumption is given.

Assumption 3.1. The nominal uncoupled continuous-time and digital subsystems $\dot{x}_{c}^{*}(t)=$ $A_{c}(t) x_{c}^{*}(t)$ and $x_{d}^{*}[k+1]=A_{d}[k] x_{d}^{*}[k]$ are both exponentially stable, that is, there exist normdependent real constants $K_{c} \geq 1$ and $K_{d} \geq 1$ such that $\left\|\Psi_{c}\left(t_{2}, t_{1}\right)\right\| \leq K_{c} e^{-a_{c}\left(t_{2}-t_{1}\right)}$ and $\left\|\Psi_{d}\left(k_{2}, k_{1}\right)\right\| \leq$ $K_{d} a_{d}^{k_{2}-k_{1}}$ for some real constants $a_{c}>0$ and $a_{d} \in[0,1)$ where $\Psi_{c}(\cdot, \cdot)$ and $\Psi_{d}[\cdot, \cdot]$ are the statetransition matrices of the uncoupled continuous-time and digital subsystems in $\Sigma$ (i.e., $\dot{\Psi}_{c}(t, 0)=$ $A_{c}(t) \Psi_{c}(t, 0) ; \Psi_{c}(0,0)=I_{n_{c}}$ with $\Psi_{c}\left(k_{2} T, k_{1} T\right)=\prod_{j=k_{1}}^{j=k_{2}-1} \Phi[j]$ between two sampling instants and $\Psi_{d}\left(k_{2}, k_{1}\right)=\prod_{j=k_{1}}^{j=k_{2}-1} A_{d}[j]$ with $\Psi_{d}[0,0]=I_{n_{d}}$ for all $t \geq 0$, any real $t_{2} \geq t_{1} \geq 0$ and any integers $k_{2} \geq k_{1} \geq 0$ ).

The following stability result holds for the nominal extended system (i.e., $\delta \equiv 0$ in

Proposition 3.2. Define

$$
\rho_{k} \operatorname{Max}\left(\operatorname{Max}_{1 \leq i \leq n_{c}} \sum_{j=1}^{n_{c}} \int_{0}^{T}\left|\Psi_{c}^{(i j)}((k+1) T, k T+\tau) A_{c d}(\tau) d \tau\right|\right), \quad \operatorname{Max}_{1 \leq i \leq n_{d}}\left(\sum_{j=1}^{n_{d}}\left|A_{d c}^{(i j)}[k]\right|\right) .
$$

Thus, the nominal extended discrete system is exponentially stable if Assumption 3.1 holds and $\rho_{k}<$ $1-\operatorname{Max}\left(e^{-a_{c} T}, a_{d}\right)$ for all integer $k \geq 0$.

Proof. Decompose $A[k]=A_{0}[k]+\tilde{A}[k]$ in (2.11) with $A_{0}[k]=\operatorname{Block} \operatorname{Diag}\left(\Phi_{c}[k], A_{d}[k]\right)$, $\Phi_{c}[k]=\Psi_{c}((k+1) T, k T)$ and $A_{d}[k]=\Psi_{d}[(k+1) T, k]$ being the one sampling period $k$ th transition matrices. Thus, $x^{*}[k+1]=A[k] x^{*}[k]$ is exponentially stable if there exist real constants $\bar{K} \geq 1$ (being norm-dependent) and $\bar{a} \in[0,1)$ such that its state transition matrix $\Psi\left[k_{2}, k_{1}\right]=\prod_{j=k_{1}}^{k_{2}-1} A[j]$ satisfies $\left\|\Psi\left[k_{2}, k_{1}\right]\right\| \leq \bar{K} \bar{a}^{k_{2}-k_{1}}, \bar{K}=1$ for the $1_{2}$-matrix norm given by the maximum modulus within the whole set of eigenvalues. Also, $\rho_{k}=\|\tilde{A}[k]\|_{2}$, from 
the definition of $\rho_{k}$ and $\tilde{A}[k]=A[k]-A_{0}[k]$, lies in the union $\bigcup_{i=1}^{n_{c}} R_{i}$ of the discs $R_{i}=$ $\left\{z:|z| \leq \sum_{j=1}^{n_{c}+n_{d}}\left|\tilde{A}^{(i j)}[k]\right|\right\}$ from Gerschgorin's circle theorem, [17]. Therefore, $\|A[k]\|_{2} \leq$ $\operatorname{Max}\left(e^{-a_{c} T}, a_{d}\right)+\rho_{k} \leq \bar{a}<1$ for all integer $k \geq 0$ if Assumption 3.1 and (3.1) hold. Thus, the nominal extended system is exponentially stable and the result has been proved.

\subsection{Stability of the Discrete Disturbed Extended System $\Sigma_{d}$}

The following result gives sufficient conditions for stability of the extended discrete system (2.10) within a closed ball of the extended state $x[\cdot]$.

Proposition 3.3. Assume that Proposition 3.2 holds under Assumption 3.1 (i.e., the nominal extended system (2.10) is exponentially stable) under the stronger condition $\|A[k]\|_{2} \leq \bar{a}<$ $1-\bar{K}_{d} / \bar{K}<1$ where the real constants $\bar{K}$ and $\bar{a}$ are related to the state transition matrix of $\Sigma_{d}$ (2.10) and defined in the proof of Proposition 3.2, and

$$
\begin{gathered}
\bar{K}_{d}=K_{c} a_{c}^{-1} \beta_{c}+K_{d} \beta_{d} \\
\beta_{h}=\beta_{h c}^{f}+\beta_{h d}^{f}+\beta_{h c}^{g}+\beta_{h d}^{g}
\end{gathered}
$$

for $h=c, d$; and $\bar{K}_{d}<1$. Thus, the state vector is uniformly bounded according to

$$
\|x[k]\| \leq\left(\bar{a}^{k}+\bar{K} \bar{K}_{d} \frac{1-\bar{a}^{k}}{1-\bar{a}}\right) r \leq r
$$

for all integer $k \geq 0$ provided that $\operatorname{Max}\left(\left\|x_{c}[0]\right\|,\left\|x_{d}[0]\right\|\right)<r / 2 \bar{K} \leq r / 2$.

Proof. First, note from direct calculus from (2.6)-(2.9) that the disturbance signal $\delta[k]$ in (2.10) satisfies

$$
\|\delta[k]\| \leq\left\|\delta_{c}^{\prime}[k]\right\|+\left\|\delta_{d}[k]\right\| \leq \bar{K}_{d} r
$$

provided that $\operatorname{Max}\left(\left\|x_{c}[k]\right\|,\left\|x_{d}[k]\right\|\right)<r / 2$ for all integer $k \geq 0$ since $\bar{K}>1$ and $\bar{a}<1-$ $\bar{K}_{d} / \bar{K}$ imply $\bar{a}+\bar{K}_{d}<1$. Consider the set of sequences $\{y[k], k \geq 0\}$ equipped with the $\ell_{\infty}$ norm for sequences $\|y\|_{\infty}=\operatorname{Max}_{0 \leq k \leq \infty}(\|y[k]\|)$. Thus, the operator $\mathbf{T}_{\mathbf{d}}$ defined by $\left(\mathbf{T}_{\mathrm{d}} y\right)[k]=$ $A[k] y[k]+\delta[k]$ is a contraction on the closed subset $\mathbb{R}_{\mathbf{d}}$ of bounded $n_{d}$-vector sequences $\left\{y[k], k \geq 0:\|y\|_{\infty} \leq r\right\}$. By the contraction mapping theorem $[17,18]$, there is a unique solution $y[k+1]=\left(\mathbf{T}_{\mathbf{d}} y\right)[k]$ (fixed point) with sequences in $\mathbb{R}_{\mathbf{d}}$, and

$$
\|x[k]\|=\left\|\Psi(k, 0) x[0]+\sum_{i=0}^{k-1} \Psi(k, i+1) \delta[i]\right\| \leq \bar{K} \bar{a}^{k}\|x[0]\|+\bar{K} \bar{K}_{d} r\left(\sum_{i=0}^{k-1} \bar{a}^{i}\right),
$$


which leads directly to (3.4) since $\bar{a}<1-\bar{K}_{d} / \bar{K}<1$ implies

$$
\sum_{i=0}^{k-1} \bar{a}^{i}=\frac{1-\bar{a}^{k}}{1-\bar{a}} ; \quad \bar{a}^{k}+\bar{K}_{\bar{K}} \frac{1-\bar{a}^{k}}{1-\bar{a}} \leq 1+\bar{a}^{k}-\bar{a} \leq 1 .
$$

\subsection{Stability of the Continuous-Time Substate and State Boundedness inbetween Consecutive Sampling Instants}

Now, the solution to (2.1) subject to (2.2) and (2.3) is analyzed by taking into account that $\|x[k]\| \leq r$ provided that Proposition 3.3 holds. A total stability argument is used as main tool for the proof of stability of the continuous-time subsystem.

Proposition 3.4. Assume that Proposition 3.3 holds, $\operatorname{Sup}_{0 \leq t<\infty}\left(\left\|A_{c d}(t)\right\|\right) \leq a_{c d},\left\|x_{c}(0)\right\| \leq r / 2 K_{c}$ and $K_{c} \bar{K}_{c} / a_{c}<1$, where

$$
\bar{K}_{c}=K_{c}^{f}+K_{c}^{g} ; \quad K_{c}^{f}=\beta_{c c}^{f}+\beta_{c d}^{f} ; \quad K_{c}^{g}=a_{c d}+\beta_{c c}^{f}+\beta_{c d}^{g} .
$$

Thus, there is a unique solution $x_{c}(t)$ to (2.1) such that for all $t \geq 0$ :

$$
\left\|x_{c}(t)\right\| \leq K_{c} e^{-\left(a_{c}-K_{c} K_{c}^{f}\right) t}\left\|x_{0}\right\|+\frac{K_{c} K_{c}^{g}}{a_{c}-K_{c} K_{c}^{f}} r\left(1-e^{-\left(a_{c}-K_{c} K_{c}^{f}\right) t}\right) \leq r .
$$

Proof. One gets directly from (2.1),

$$
x_{c}(t)=\Psi_{c}(t, 0) x_{c}(0)+\int_{0}^{t} \Psi_{c}(t, \tau) \delta_{c}^{0}(\tau) d \tau
$$

with $x_{c}(0)=x_{c}[0]$ and $\delta_{c}^{0}(t)=A_{c d}(t) x_{d}[k]+\delta_{c}(t)$. Under the set of constraints $C,\left\|\delta_{c}^{0}(t)\right\| \leq$ $\bar{K}_{c} r$ for all $t \geq 0$ subject to (3.8). Using similar arguments as in the proof of Proposition 3.3, consider the Banach space $\mathbf{B}_{\mathbf{c}}=C[0, \infty)$ of continuous, bounded $n_{c}$-vector sequences defined on $[0, \infty)$, and equipped with the $L_{\infty}$-norm $\|y\|_{\infty}=\operatorname{Sup}_{0 \leq t \leq \infty}(\|y(t)\|)$. The operator $\mathbf{T}_{\mathbf{c}}$ is defined:

$$
\left(\mathrm{T}_{\mathrm{c}} y\right)(t)=\Psi_{c}(t, 0) x_{0}+\int_{0}^{t} \Psi_{c}(t, \tau) \delta_{c}^{0}(\tau) d \tau
$$

is a contraction of the closed subset $\mathbb{R}_{\mathbf{c}}=\left\{y \in \mathbf{B}_{\mathbf{c}}:\|y\|_{\infty} \leq r\right\}$ of $\mathbf{B}_{\mathbf{c}}$, because for $\left\|y_{i}\right\|_{\infty} \leq r \quad(i=$ $1,2)$, one gets from (3.8)-(3.11) that

$$
\begin{aligned}
& \left\|\mathrm{T}_{\mathrm{c}} y(t)\right\|_{\infty} \leq K_{\mathrm{c}}\left\{e^{-a_{c} t}\left\|x_{c}(0)\right\|+\frac{\bar{K}_{c}}{a_{c}}\left(1-e^{-a_{c} t}\right) r\right\} \leq r, \\
& \Longrightarrow\left\|\mathrm{T}_{\mathrm{c}} y_{1}-\mathrm{T}_{\mathrm{c}} y_{2}\right\|_{\infty} \leq \bar{K}_{\mathrm{c}} a_{c}^{-1}\left\|y_{1}-y_{2}\right\|_{\infty} \leq\left\|y_{1}-y_{2}\right\|_{\infty},
\end{aligned}
$$


for $\left\|x_{c}(0)\right\|=\left\|x_{c}[0]\right\| \leq r / 2 K_{c} \leq r / 2 \bar{K} \leq r$ since $\left\|x_{d}[k]\right\| \leq r / 2 \bar{K} \leq r$ for all $k \geq 0$ from Proposition 3.3. By the contraction mapping theorem, $[17,18]$, there exists a unique solution of (3.11) in $\mathbb{R}_{\mathbf{c}}$, the fixed point of $\mathbf{T}_{\mathbf{c}}$. Thus, one gets from (3.11) that

$$
\|x(t)\| \leq K_{c}\left\{e^{-a_{c} t}\left\|x_{0}\right\|+K_{c}^{f} \int_{0}^{t} e^{-a_{c}(t-\tau)}\left\|x_{c}(\tau)\right\| d \tau+K_{c}^{g} r \int_{0}^{t} e^{-a_{c}(t-\tau)} d \tau\right\}
$$

which leads to (3.9) from Bellman-Gronwall lemma [18].

Remark 3.5 (Combined interpretation of Propositions 3.2-3.4). Assumption 3.1 and Propositions 3.2-3.4 yield the following robust stability conditions for the system $\Sigma$ by using $1_{2}$ vector and matrix norms, that is, $K_{c}=K_{d}=1$, provided that $\left\|x_{c}[0]\right\| \leq r / 2$ and $\left\|x_{d}[0]\right\| \leq r / 2$ :

$$
\begin{aligned}
& \rho^{*}+\rho+\beta_{c} a_{c}^{-1}+\beta_{d}<1 ; \quad \bar{K}_{\mathrm{c}} a_{c}^{-1}<1, \\
& \rho^{*}=\operatorname{Max}_{0 \leq k \leq \infty}\left(e^{-a_{c} T}, a_{d}\right) ; \quad \rho=\operatorname{Max}_{0 \leq k \leq \infty}\left(\rho_{k}\right)
\end{aligned}
$$

with $\beta_{c}$ and $\beta_{d}$ being real constants defined in (3.3) related to the set of constraints $C, \rho_{k}$ and $\bar{K}_{c}$ defined in (2.12) and (3.8). In particular, (a) $\rho^{*}<1$ guarantees the exponential stability of the uncoupled nominal continuous-time and digital subsystems (i.e., $\delta_{c} \equiv 0, \delta_{d} \equiv 0$ ), (b) $\rho^{*}+\rho<1$ guarantees that the exponential stability is not destroyed in the nominal extended system $\Sigma_{d}^{*}$ by the existence of linear couplings between the continuous-time and digital substates, (c) the first inequality in (3.14) guarantees that the state disturbances in $\Sigma$ are sufficiently small in terms of the real constants defining their overbounding functions while satisfying $C$ so that the extended discrete system $\Sigma_{d}$ maintains the stability of its nominal description $\Sigma_{d}^{*}$. If, furthermore, the second constraint of (3.14) holds then the signal boundedness is guaranteed inbetween sampling instants according to (3.9) and the overall hybrid system $\Sigma$ is robustly stable.

\subsection{Some Links with Singular Perturbation Theory}

In some particular descriptions within the class $\Sigma$, the perturbation theory can be combined with the above analysis. Assume, for instance, that the linear dynamics of $\Sigma$ is subject to variations defined by a small parameter $\varepsilon, A_{d c}$ and $A_{d d}$ are time-invariant, and $A_{c}(t)=\varepsilon A_{c}$ for all $t \geq 0$ and $A_{c d}(t)=\rho(\varepsilon) e^{\varepsilon A_{c} t}$ with $\rho(\varepsilon) \leq \bar{\rho}<\infty$ for all $\varepsilon \in\left[0, \varepsilon^{*}\right)$. Thus, a direct series expansion around $\varepsilon T$ of the state transition matrix of the continuous subsystem yields

$$
\begin{aligned}
\Psi_{c}((k+1) T, k T) & =e^{\varepsilon A_{c} T}=I_{n_{c}}+\varepsilon A_{c} T+\Delta\left(\varepsilon, T, A_{c}\right) \\
\int_{k T}^{(k+1) T} \Psi_{c}((k+1) T) A_{c d}(\tau) d \tau & =\left(I_{n}+\varepsilon A_{c} T+\Delta\left(\varepsilon, A_{c}, T\right)\right) \int_{k T}^{(k+1) T} e^{-\varepsilon A_{c} \tau} A_{c d}(\tau) d \tau \\
& =\left(\varepsilon A_{c}+\rho(\varepsilon)\left[I_{n_{c}}+\Delta\left(\varepsilon, T, A_{c}\right)\right]\right) T .
\end{aligned}
$$


Note that $\left\|\Delta\left(\varepsilon, T, A_{c}\right)\right\|_{2} \leq 1+\varepsilon A_{c} T+\varepsilon T\left|\lambda_{\max }\left(A_{c}\right)\right|=\delta(\varepsilon) \leq \bar{\delta}<\infty$ for all $\varepsilon \in\left[0, \varepsilon^{*}\right)$ and $\rho(\varepsilon) T\left[I_{n_{c}}+\Delta\left(\varepsilon, T, A_{c}\right)\right] \leq \bar{\rho} T(1+\bar{\delta})<\infty$ for all $\varepsilon \in\left[0, \varepsilon^{*}\right)$. Thus,

$$
\begin{aligned}
A(\varepsilon) & =A^{*}(\varepsilon)+\Delta \bar{A}(\varepsilon) ; \quad A^{*}(\varepsilon)=\left\lfloor\begin{array}{cc}
I_{n_{c}}+\varepsilon A_{c} T & \varepsilon A_{c} T \\
A_{d c} & A_{d}
\end{array}\right] ; \\
\Delta \bar{A}(\varepsilon) & =\left[\begin{array}{cc}
\Delta\left(\varepsilon, T, A_{c}\right) & \rho(\varepsilon) T\left(I_{n_{c}}+\Delta\left(\varepsilon, T, A_{c}\right)\right) \\
0 & 0
\end{array}\right]
\end{aligned}
$$

is time-invariant in (2.10). Thus, the discrete system $\Sigma_{d}$ of (2.10) satisfies equivalently

$$
z[k+1]=\widehat{A}^{*}(\varepsilon) z[k]+(\delta[k]+\Delta \bar{A}(\varepsilon) z[k])
$$

by defining (see [1])

$$
\widehat{A}^{*}(\varepsilon)=\widehat{I}+\widehat{A}_{21}+\widehat{A}_{22}+\varepsilon\left(\widehat{A}_{11}+\widehat{A}_{12}\right) \quad \text { for } \varepsilon \in\left[0, \varepsilon^{*}\right)
$$

through the extended $n=n_{c}+n_{d}$-matrices

$$
\begin{gathered}
\widehat{A}_{11}=\left[\begin{array}{cc}
T A_{c} & 0 \\
0 & 0
\end{array}\right] ; \quad \widehat{A}_{12}=\left[\begin{array}{cc}
0 & T A_{c} \\
0 & 0
\end{array}\right] ; \quad \widehat{I}=\left[\begin{array}{cc}
\widehat{I}_{n_{c}} & 0 \\
0 & 0
\end{array}\right], \\
\widehat{A}_{21}=\left[\begin{array}{cc}
0 & 0 \\
A_{d c} & 0
\end{array}\right] ; \quad \widehat{A}_{22}=\left[\begin{array}{cc}
0 & 0 \\
0 & A_{d}
\end{array}\right] .
\end{gathered}
$$

Note that Schur's stability of $\widehat{A}^{*}(\varepsilon)$ is equivalent to exponential stability of the unforced timeinvariant system $\Sigma_{d}(\varepsilon): z^{*}[k+1]=\widehat{A}^{*}(\varepsilon) z^{*}[k]$ since $\widehat{A}^{*}(\varepsilon)$ has its eigenvalues in $|z|<1$ for all $\varepsilon \in\left[0, \varepsilon^{*}\right)$. Thus, the subsequent result follows directly from Proposition 3.3 by using a previous result in [1].

Proposition 3.6. Define $v(\varepsilon):=v(\widehat{A}(\varepsilon))=\operatorname{det}(\widehat{A}(\varepsilon) \otimes \widehat{A}(\varepsilon)-\widehat{I} \otimes \widehat{I})$, where " $\otimes "$ denotes the direct Kronecker product of matrices which is a matrix of order $4 n^{2}$. Thus, the following items hold.

(i) If $v(\varepsilon)$ has no positive zeros, then either $\widehat{A}(\varepsilon)$ is Schur stable for all $\varepsilon>0$ or it is not Schur stable for any $\varepsilon>0$.

(ii) If $v(\varepsilon)$ has positive zeros, let $\bar{\varepsilon}$ be the smallest such zero. If $\widehat{A}\left(\varepsilon_{1}\right)$ is Schur stable for any $\varepsilon_{1} \in[0, \bar{\varepsilon})$, then $\varepsilon^{*}=\bar{\varepsilon}$. Otherwise, $\widehat{A}(\varepsilon)$ is not Schur stable for all sufficiently small and positive values of $\varepsilon$.

(iii) The extended discrete system $\Sigma_{d}(\bar{\varepsilon})$ is stable for all $\varepsilon \in\left[0, \varepsilon^{*}\right)$ satisfying $\operatorname{Max}\left(\left\|x_{c}[0], x_{d}[0]\right\|\right) \leq r / 2 \bar{K}$ and $\left\|A^{*}(\varepsilon)\right\|_{2}+\bar{K}_{d}+\operatorname{Max}_{0 \leq \varepsilon \leq \varepsilon^{*}}\left(\|\Delta A(\varepsilon)\|_{2}\right)<1$ with $\bar{K}_{d}$ defined in (3.2). 


\section{Numerical Example}

The following third-order system (so-called controlled plant), whose state-space description lie within the class of hybrid system (2.1), is considered:

$$
\begin{gathered}
\ddot{y}(t)+a_{1} \dot{y}(t)+a_{2} y(t)+a_{3} y[k]+4 y[k-1] \\
=b_{0} u(t)+b_{1} \dot{u}(t)+b_{2} u[k]+3 u[k-1]+0.3(z[k]+\delta(t)), \\
z[k+1]=0.2 z[k]+1.1 u[k]+1.3 y[k], \\
\dot{\delta}(t)=-7 \delta(t)+8.5 u(t),
\end{gathered}
$$

for all $t \in[k T,(k+1) T)$ and any integer $k \in \mathbb{Z}_{0_{+}}$. The signal $u(t)$ is a stabilizing outputfeedback control signal generated from an hybrid controller as follows:

$$
\begin{gathered}
u(t)=\frac{G_{1}(D, q)}{L(D, q)} u(t)+\frac{G_{2}(D, q)}{L(D, q)} y(t), \\
G_{1}(D, q)=D^{2} q^{2}-q^{2} D+D^{2} q+1.25 q^{2}-D q+0.25 q-1.44187 D^{2}+0.206426 D-2.54251, \\
G_{2}(D, q)=1.12792\left(D^{2} q^{2}-0.269774 q^{2} D+1.10629\right), \\
L(D, q)=(D-0.5)^{2}(q+0.5)^{2},
\end{gathered}
$$

where $q$, defined by $q v(t)=v(t+T)$ for any real vector function $v: \mathbb{R}_{0+} \rightarrow \mathbb{R}^{p}$, is the discrete one-step advance operator and $D:=d / d t$ is the time-derivative operator. After substituting the control law in the plant description, the resulting closed-loop system is of the general form given while driven only by the disturbance $\delta(t)$. The signal $\delta(t)=\delta_{c}(t)$ is a perturbation which satisfies the general assumptions-constraints $C$ of the theory of total stability. There are six parameters to be estimated by the estimation schemes: $a_{1}=-1, a_{2}=2, a_{3}=3, b_{0}=$ $1, b_{1}=b_{2}=2$, and $b_{3}=3$. The constant sampling period is $T=0.4$. Finally, the reference model is a third-order highly damped one of discrete regulation. The plant output (i.e., the solution of (4.1)) is shown in Figure 1.

Note that both the extended discrete system and the continuous one are Lyapunov stable since the output is bounded for all time. Remark 3.5, which is a combined interpretation of Propositions 3.2-3.4, holds with all the relevant functions in the control scheme which are uniformly bounded for all time, that is, "at" and "in-between" sampling instants. If the disturbance $\delta(t)$ is zeroed, then the closed-loop system is globally asymptotically stable.

\section{Generalizations to Hybrid Systems with Time-Varying Sampling Periods}

The more general case when the sequence of sampling periods is, in general, not constant and is discussed in the following by using fixed point theory. The main mathematical result is concentrated in Theorem 5.1 which also contains many of the above results as particular case. Some corollaries to Theorem 5.1 are also given. Equations (2.1)-(2.5) are now assumed 


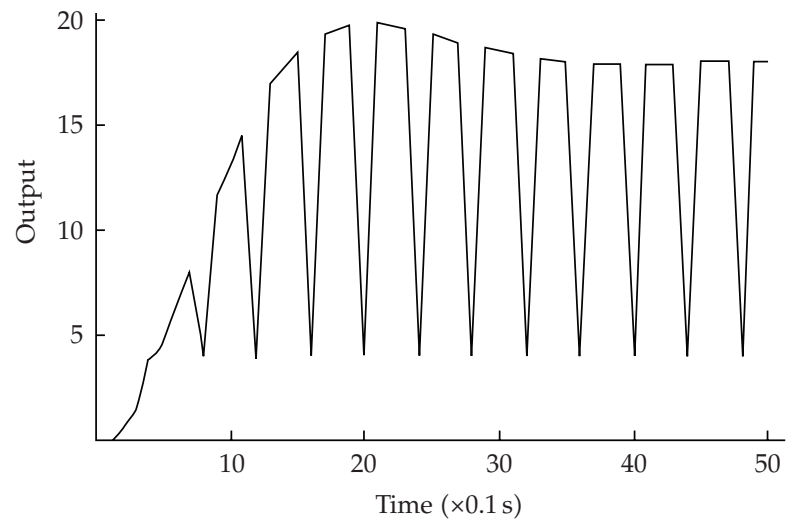

Figure 1: Output versus time.

to run for all time $t \in\left[t_{k}, t_{k+1}\right), k \in \mathbb{Z}_{0+}$, where $\left\{t_{k}\right\}_{k \in \mathbb{Z}_{0}^{+}}$and $\left\{T_{k}\right\}_{k \in \mathbb{Z}_{0}^{+}}$are the sequences of sampling instants and sampling periods (i.e., interval lengths inbetween two consecutive sampling instants), respectively, with $t_{k}=\sum_{j=0}^{k-1} T_{j}, t_{0}=0$, for all $k \in \mathbb{Z}_{+}$discrete time integer index $k \in \mathbb{Z}_{0+}$. The following result holds.

Theorem 5.1. Assume that constraints $C$ hold, $\left\{t_{k}\right\}_{k \in \mathbb{Z}_{0+}}$ is a real monotone strictly increasing sequence of sampling instants and $\left\{T_{k}\right\}_{k \in \mathbb{Z}_{0+}}$ is the real sequence of sampling periods with $\mathbb{R}_{+} \ni T_{k}:=$ $t_{k+1}-t_{k} \in\left[\varepsilon_{T}, \bar{T}\right] \subset(0, \infty)$ for some constants $\varepsilon_{T}, \bar{T}\left(\geq \varepsilon_{T}\right) \in \mathbb{R}_{+}$. Then, the following properties hold:

(i) Assume that $\operatorname{Max}\left(\left\|x_{c}[0]\right\|,\left\|x_{d}[0]\right\|\right) \leq r, A_{c}$ is a stability matrix satisfying $a_{c}>K_{c}\left(\beta_{c c}^{f}+\right.$ $\left.\beta_{c d}^{f}\right), \beta_{c c}^{g}=\beta_{c d}^{g}=0,\{A[k]\}_{k \in \mathbb{Z}_{0+}}$ is a real sequence of convergent (or Schur) matrices, and the real sequence $\left\{a_{c}\left\|\bar{\Psi}_{c}\left(t_{k}+\tau, t_{k}\right)\right\| /\left(a_{c}-\left(\beta_{c c}^{f}+\beta_{c d}^{f}\right) K_{c}\right)\right\}_{k \in \mathbb{Z}_{0+}}$ has all its elements not greater than unity. Then, the state trajectory solution $\left(x_{c}^{T}(t), x_{d}^{T}[k]\right)^{T}: \mathbb{R}_{0_{+}} \times \mathbb{R}^{n_{c}} \times$ $\mathbb{Z}_{0+} \mathbb{R}^{n_{d}} \rightarrow \mathbb{R}^{n}$ is in the closed ball $\bar{B}(0, r):=\left\{x \in \mathbb{R}^{n}:\|x\| \leq r\right\} \subset \mathbb{R}^{n}$ centred at zero; for all $(t, k) \in \mathbb{R}_{0+} \times \mathbb{Z}_{0+}$. Thus, it is totally stable within such a ball.

(ii) Assume that $\operatorname{Max}\left(\left\|x_{c}[0]\right\|,\left\|x_{d}[0]\right\|\right) \leq r$. Then, the state trajectory solution $\left(x_{c}^{T}(t), x_{d}^{T}[k]\right)^{T}: \mathbb{R}_{0+} \times \mathbb{R}^{n_{c}} \times \mathbb{Z}_{0+} \times \mathbb{R}^{n_{d}} \rightarrow \mathbb{R}^{n}$ is totally stable within the closed ball $\bar{B}(0, r):=\left\{x \in \mathbb{R}^{n}:\|x\| \leq r\right\} \subset \mathbb{R}^{n}$ centred at zero; for all $(t, k) \in \mathbb{R}_{0+} \times \mathbb{Z}_{0+}$ if

$\bar{\rho}\left(1+\frac{\left(\beta_{c c}^{f}+\beta_{c d}^{f}\right) \rho_{c} \bar{T}}{1-\bar{\rho}}+\beta_{d c}^{f}+\beta_{d d}^{f}\right)+\frac{1}{1-\bar{\rho}}\left[\left(\beta_{c c}^{g}+\beta_{c d}^{g}\right) \rho_{c} \bar{T}+\beta_{d c}^{g}+\beta_{d d}^{g}\right] \leq 1$,

for all $k \in \mathbb{Z}_{+}$, where $\rho$ is an upper-bound of the sequence $\left\{\left\|\Psi\left(t_{k}, 0\right)\right\|\right\}_{k \in \mathbb{Z}_{0_{+}}}$with $\Psi\left(t_{k}, 0\right):=\prod_{i=0}^{k-1}[A[i]]$ provided that $1>\rho \geq \max _{k} \in \mathbb{Z}_{+}\left\|\Psi\left(t_{k}, 0\right)\right\|, 1>\rho_{c} \geq$ $\max _{k \in \mathbb{Z}_{+}}\left\|\Psi_{c}\left(t_{k}, k_{k-1}\right)\right\| \geq K_{c} / a_{c}$ or, if $\bar{\rho} \leq 1$, provided that $\beta_{c c}^{f}=\beta_{c d}^{f}=\beta_{d c}^{f}=\beta_{d d}^{f}=$ $\beta_{c c}^{g}=\beta_{c d}^{g}=\beta_{d c}^{g}=\beta_{d d}^{g}=0$ (i.e., in case of absence of perturbations).

(iii) If $\rho+\beta_{d c}^{f}+\beta_{d d}^{f}<1$ and $\beta_{c c}^{f}=\beta_{c d}^{f}=\beta_{d c}^{f}=\beta_{d d}^{f}=\beta_{c c}^{g}=\beta_{c d}^{g}=\beta_{d c}^{g}=\beta_{d d}^{g}=0$, then 
(a) the bounded sequence $\{x[k]\}_{k \in \mathbb{Z}_{0}^{+}}$has a unique fixed point $x_{0}^{*}$ in the convex closed ball $\bar{B}(0, r)$ so that $x[k] \rightarrow x^{*}(0)=\left(x_{c}^{*^{T}}(0), x_{d}^{*^{T}}\right)^{T}$ as $\mathbb{Z}_{0+} \ni k \rightarrow \infty$ for any $\operatorname{Max}\left(\left\|x_{c}[0]\right\|,\left\|x_{d}[0]\right\|\right) \leq r$,

(b) Assume that $T_{k} \rightarrow T \in\left(\varepsilon_{T}, \bar{T}\right)$ as $\mathbb{Z}_{0+} \ni k \rightarrow \infty$. Then, the whole state trajectory solution $\left(x_{c}^{T}(t), x_{d}^{T}[k]\right)^{T}: \mathbb{R}_{0_{+}} \times \mathbb{R}^{n_{c}} \times \mathbb{Z}_{0+} \times \mathbb{R}^{n_{d}} \rightarrow \mathbb{R}^{n}$ has a fixed point $x^{*}(\tau)=$ $\left(x_{c}^{*^{T}}(\tau), x_{d}^{*^{T}}\right)^{T} \in \bar{B}(0, r)$ for each $\tau \in[0, T)$.

(iv) Assume that the constraints C4 are modified as follows:

$$
\begin{gathered}
C 4^{\prime}: g_{c c}\left(t, x_{c i}\right) \leq \beta_{c c}^{g} x_{c i} ; \quad g_{c d}\left(t, x_{d i}\right) \leq \beta_{c d}^{g} x_{d i}, \quad g_{d c}\left(t, x_{c i}\right) \leq \beta_{d c}^{g} x_{c i} ; \\
g_{d d}\left(t, x_{d i}\right) \leq \beta_{d d}^{g} x_{d i} ; \quad i=1,2 .
\end{gathered}
$$

Then, a unique fixed point $z^{*} \in \bar{B}(0, r)$ exists for the whole state trajectory solution $\left(x_{c}^{T}(t), x_{d}^{T}[k]\right)^{T}$ : $\mathbb{R}_{0+} \times \mathbb{R}^{n_{c}} \times \mathbb{Z}_{0+} \times \mathbb{R}^{n_{d}} \rightarrow \mathbb{R}^{n}$ provided that the following constraint holds:

$$
\rho+\beta_{d c}^{f}+\beta_{d d}^{f}+\rho_{c} \bar{T}\left(\beta_{c c}^{f}+\beta_{c d}^{f}+\beta_{c c}^{g}+\beta_{c d}^{g}\right)+\beta_{d c}^{g}+\beta_{d d}^{g}<1
$$

For sufficiently small $\left(\beta_{d c}^{f}+\beta_{d d}^{f}+\beta_{d c}^{f}+\beta_{c d}^{g}\right)$ and any given $\rho<1$, there exists a sufficiently small upper-bound of the sequence of sampling periods $\bar{T}$ such that the above constraint holds for a given constant $\left(\beta_{c c}^{f}+\beta_{c d}^{f}+\beta_{c c}^{g}+\beta_{c d}^{g}\right)$.

Proof. (i) One gets directly from (3.10) via (2.10) that

$$
\begin{aligned}
x\left(t_{k}+\tau\right) & :=\left(x_{c}^{T}\left(t_{k}+\tau\right), x_{d}^{T}[k]\right)^{T} \\
& =\Psi_{c}\left(t_{k}+\tau, t_{k}\right) x_{c}\left(t_{k}\right)+\int_{0}^{\tau} \Psi_{c}\left(t_{k}+\tau, t_{k}+s\right) \delta_{c}^{0}\left(t_{k}+s\right) d s \\
& =\Psi_{c}\left(t_{k}+\tau, t_{k}\right)\left(E_{1} x[k]+\int_{0}^{\tau} \Psi_{c}\left(t_{k}+\tau, t_{k}+s\right) \delta_{c}^{0}\left(t_{k}+s\right) d s\right) \\
& =\bar{\Psi}_{c}\left(t_{k}+\tau, t_{k}\right) x[k]+\int_{0}^{\tau} \Psi_{c}\left(t_{k}+\tau, t_{k}+s\right) \delta_{c}\left(t_{k}+s\right) d s,
\end{aligned}
$$

where

$$
\bar{\Psi}_{c}\left(t_{k}+\tau, t_{k}\right):=\Psi_{c}\left(t_{k}+\tau, t_{k}\right)\left(E_{1}+\left(\left(\int_{0}^{\tau} \Psi_{c}\left(t_{k}+\tau, t_{k}+s\right) A_{c d}\left(t_{k}+s\right) d s\right) E_{2}\right)\right)
$$

and $E_{1}:=\operatorname{Diag}\left(I_{n_{c}}, 0_{n_{d}}\right), E_{2}:=\operatorname{Diag}\left(0_{n_{c}}, I_{n_{d}}\right)=I_{n}-E_{1}$; for all $\tau \in\left[0, T_{k}\right)$, for all $k \in \mathbb{Z}_{0+}$. Since the threshold $\varepsilon_{T}$ for the minimum sampling period between any two consecutive 
samples exist, the state trajectory solution of (2.1)-(2.4) is unique for each given bounded initial condition. Then, for any two state-trajectory solutions $\bar{x}, \bar{y}: \mathbb{R}_{0}^{+} \rightarrow \mathbb{R}^{n}$ of (5.4):

$$
\begin{aligned}
\left\|x\left(t_{k}+\tau\right)-y\left(t_{k}+\tau\right)\right\| \leq & \left\|\bar{\Psi}_{c}\left(t_{k}+\tau, t_{k}\right)\right\|\|x[k]-y[k]\| \\
& +\int_{0}^{\tau}\left\|\Psi_{c}\left(t_{k}+\tau, t_{k}+s\right)\right\|\left\|\delta_{c x}\left(t_{k}+s\right)-\delta_{c y}\left(t_{k}+s\right)\right\| d s \\
\leq & \left\|\bar{\Psi}_{c}\left(t_{k}+\tau, t_{k}\right)\right\|\|x[k]-y[k]\| \\
& +\left(\beta_{c c}^{f}+\beta_{c d}^{f}\right) \int_{0}^{\tau}\left\|\Psi_{c}\left(t_{k}+\tau, t_{k}+s\right)\right\|\left\|x_{c x}\left(t_{k}+s\right)-x_{c y}\left(t_{k}+s\right)\right\| d s \\
& +2\left(\beta_{c c}^{g}+\beta_{c d}^{g}\right) r\left(\int_{0}^{\tau}\left\|\Psi_{c}\left(t_{k}+\tau, t_{k}+s\right)\right\| d s\right) \\
\leq & \left\|\bar{\Psi}_{c}\left(t_{k}+\tau, t_{k}\right)\right\|\|x[k]-y[k]\|+\frac{K_{c}}{a_{c}}\left(1-e^{-a_{c} \tau}\right)\left(\beta_{c c}^{f}+\beta_{c d}^{f}\right) \\
& \times{ }_{t_{k} \leq s \leq t_{k+1}} \operatorname{Max}_{c x}(s)-x_{c y}(s) \| \\
& +2 \frac{K_{c}}{a_{c}}\left(1-e^{-a_{c} \tau}\right)\left(\beta_{c c}^{g}+\beta_{c d}^{g}\right) r ; \quad \forall \tau \in\left[0, T_{k}\right) .
\end{aligned}
$$

If $a_{c}>K_{c}\left(\beta_{c c}^{f}+\beta_{c d}^{f}\right)$, then one deduces from (5.6) that

$$
\begin{array}{r}
\left\|x\left(t_{k}+\tau\right)-y\left(t_{k}+\tau\right)\right\| \\
\leq\left(1-\frac{K_{c}}{a_{c}}\left(\beta_{c c}^{f}+\beta_{c d}^{f}\right)\right)^{-1}\left(\left\|\bar{\Psi}_{c}\left(t_{k}+\tau, t_{k}\right)\right\|\|x[k]-y[k]\|+2 \frac{K_{c}}{a_{c}}\left(\beta_{c c}^{g}+\beta_{c d}^{g}\right) r\right) \\
\forall k \in \mathbb{Z}_{0+}, \quad \forall \tau \in\left[0, T_{k}\right) \\
\leq \frac{a_{c}}{a_{c}-\left(\beta_{c c}^{f}+\beta_{c d}^{f}\right) K_{c}}\left(\left\|\bar{\Psi}_{c}\left(t_{k}+\tau, t_{k}\right)\right\|\|x[k]-y[k]\|+2 \frac{K_{c}}{a_{c}}\left(\beta_{c c}^{g}+\beta_{c d}^{g}\right) r\right) \\
\quad \forall k \in \mathbb{Z}_{0+,}, \quad \forall \tau \in\left[0, T_{k}\right) \\
\leq \frac{a_{c}}{a_{c}-\left(\beta_{c c}^{f}+\beta_{c d}^{f}\right) K_{c}}\left(\left\|\bar{\Psi}_{c}\left(t_{k}+\tau, t_{k}\right)\right\|\|x[k]-y[k]\|+2 \frac{K_{c}}{a_{c}}\left(\beta_{c c}^{g}+\beta_{c d}^{g}\right) r\right) \\
\forall k \in \mathbb{Z}_{0+}, \quad \forall \tau \in\left[0, T_{k}\right) .
\end{array}
$$

On the other hand the combination of (3.6) and (2.12) when extended to the case of time varying-sampling leads to the following constraints at sampling instants since 
$\Phi_{c}[k]=\Psi_{c}((k+1) T, k T):$

$$
\begin{aligned}
\|x[k]-y[k]\|= & \Psi\left(t_{k}, 0\right)(x[0]-y[0])+\sum_{i=0}^{k-1} \Psi\left[t_{k}, t_{i+1}\right]\left(\delta_{x}[i]-\delta_{y}[i]\right) \| \\
= & \| \Psi\left(t_{k}, 0\right)(x[0]-y[0])+\sum_{i=0}^{k-1} \Psi\left(t_{k}, t_{i+1}\right) \\
& \left.\times\left(\int_{0}^{T_{i}}\left(\delta_{c x}^{T}\left(t_{i}+\tau\right)-\delta_{c y}^{T}\left(t_{i}+\tau\right)\right) \Psi_{c}^{T}\left(t_{i+1}-\tau\right) d \tau,\left(\delta_{d x}^{T}[i]-\delta_{d y}^{T}[i]\right)\right)\right)^{T} \| \\
\leq & \left\|\Psi\left(t_{k}, 0\right)\right\|\|x[0]-y[0]\|+\left\|\sum_{i=0}^{k-1} \Psi\left(t_{k}, t_{i+1}\right)\right\| \\
& \times\left(\left\|\int_{0}^{T_{i}} \Psi_{c}\left(t_{i+1}, t_{i}+\tau\right)\left(\delta_{c x}\left(t_{i}+\tau\right)-\delta_{c y}\left(t_{i}+\tau\right)\right) d \tau\right\|+\left\|\delta_{d x}[i]-\delta_{d y}[i]\right\|\right) \\
\leq & \left\|\Psi\left(t_{k}, 0\right)\right\|\|x[0]-y[0]\|+\left(\beta_{c c}^{f}+\beta_{c d}^{f}\right)\left\|\sum_{i=0}^{k-1} \Psi\left(t_{k}, t_{i+1}\right)\right\| \\
& \times\left\|\int_{0}^{T_{i}} \Psi_{c}\left(t_{i+1}, t_{i}+\tau\right)\left(x_{c}\left(t_{i}+\tau\right)-y_{c}\left(t_{i}+\tau\right)\right) d \tau\right\| \\
& +\left(\beta_{d c}^{f}+\beta_{d d}^{f}\right)\left\|\sum_{i=0}^{k-1} \Psi\left(t_{k}, t_{i+1}\right)\right\|\left\|x_{d}[i]-y_{d}[i]\right\| \\
& +2 r\left\|\sum_{i=0}^{k-1} \Psi\left(t_{k}, t_{i+1}\right)\right\|\left[\left(\beta_{c c}^{g}+\beta_{c d}^{g}\right)\left\|\int_{0}^{T_{i}} \Psi_{c}\left(t_{i+1}, t_{i}+\tau\right) d \tau\right\|+\beta_{d c}^{g}+\beta_{d d}^{g}\right]
\end{aligned}
$$

with $\Psi\left(t_{k}, 0\right):=\prod_{i=0}^{k C ̧ 1}[A[i]]$. Proceed by complete induction by assuming that $\|x(t)\| \leq r$; for all $t \in\left[0, t_{k-1}\right)$ for any given initial conditions and any $k \in \mathbb{Z}_{+}$.Then, $\left\|x\left(t_{k}\right)\right\| \leq r$ from (5.10) if

$$
\begin{gathered}
\left\|\Psi\left(t_{k}, 0\right)\right\|+\left(\beta_{c c}^{f}+\beta_{c d}^{f}\right)\left\|\sum_{i=0}^{k-1} \Psi\left(t_{k}, t_{i+1}\right)\right\|\left\|\int_{0}^{T_{i}} \Psi_{c}\left(t_{i+1}, t_{i}+\tau\right) d \tau\right\|+\left(\beta_{d c}^{f}+\beta_{d d}^{f}\right)\left\|\sum_{i=0}^{k-1} \Psi\left(t_{k}, t_{i+1}\right)\right\| \\
+\left\|\sum_{i=0}^{k-1} \Psi\left(t_{k}, t_{i+1}\right)\right\|\left[\left(\beta_{c c}^{g}+\beta_{c d}^{g}\right)\left\|\int_{0}^{T_{i}} \Psi_{c}\left(t_{i+1}, t_{i}+\tau\right) d \tau\right\|+\beta_{d c}^{g}+\beta_{d d}^{g}\right] \leq 1
\end{gathered}
$$

since $\operatorname{Max}\left(\operatorname{Max}_{j(\leq k-1) \in \mathbb{Z}_{+}}\left\|x_{d}[k]\right\|, \operatorname{Max}_{\left.t \leq t_{k-1}\right) \in \mathbb{R}_{+}}\left\|x_{c}(t)-\right\|\right) \leq r$ by taking $y(t)$ as the identically zero solution on $\mathbb{R}_{0_{+}}$and also $\operatorname{Max}\left(\operatorname{Max}_{j(\leq k-1) \in \mathbb{Z}_{+}}\left\|x_{d}[k]-y_{d}[k]\right\|, \operatorname{Max}_{\left.t \leq t_{k-1}\right) \in \mathbb{R}_{+}} \| x_{c}(t)-\right.$ $\left.y_{c}(t) \|\right) \leq 2 r$ for any two solutions in $\bar{B}(0, r)$, for all $t \leq t_{k-1}$. Then, (5.8) and Property (i) holds. 
(ii) It holds if

$$
\bar{\rho}\left(1+\frac{\left(\beta_{c c}^{f}+\beta_{c d}^{f}\right) \rho_{c} \bar{T}}{1-\bar{\rho}}+\beta_{d c}^{f}+\beta_{d d}^{f}\right)+\frac{1}{1-\bar{\rho}}\left[\left(\beta_{c c}^{g}+\beta_{c d}^{g}\right) \rho_{c} \bar{T}+\beta_{d c}^{g}+\beta_{d d}^{g}\right] \leq 1,
$$

for all $k \in \mathbb{Z}_{+}$provided that $1>\rho \geq \max _{k \in \mathbb{Z}_{+}}\left\|\Psi\left(t_{k}, 0\right)\right\|, 1>\rho_{c} \geq \max _{k \in \mathbb{Z}_{+}}\left\|\Psi_{c}\left(t_{k}, t_{k-1}\right)\right\| \geq$ $K_{c} / a_{c}$, then (5.8) holds, or if $\bar{\rho} \leq 1$ provided that $\beta_{c c}^{f}=\beta_{c d}^{f}=\beta_{d c}^{f}=\beta_{d d}^{f}=\beta_{c c}^{g}=\beta_{c d}^{g}=\beta_{d c}^{g}=$ $\beta_{d d}^{g}=0$, (i.e., in case of absence of perturbations). Furthermore, there exists a real constant $M \in \mathbb{R}_{+}$, dependent on $r$, such that $\|x(t)\| \leq r+M<\infty$; for all $t \in \mathbb{R}_{0_{+}}$. This conclusion follows since the real sequence $\{\|x[k]\|\}_{k \in \mathbb{Z}_{0+}}$ is uniformly bounded for any initial conditions fulfilling $\|x[0]\| \leq r$ and the mild continuously time differentiable state trajectory solution $x_{c}(t)$ cannot be unbounded on any open finite-time interval $\left(t_{k}, t_{k+1}\right)$; for all $k \in \mathbb{Z}_{0+}$ since $\left\|x_{c}\left(t_{k}\right)\right\| \leq\|x[k]\| \leq r$.

(iii) The inequality (5.10) adopts the following particular form at $t=t_{k+1}$ by taking initial conditions at $t=t_{k}$ :

$$
\begin{aligned}
&\|x[k+1]-y[k+1]\| \\
& \leq\left\|\Psi\left(t_{k+1}, t_{k}\right)\right\|\|x[k]-y[k]\|+\left(\beta_{c c}^{f}+\beta_{c d}^{f}\right) \\
& \times\left\|\int_{0}^{T_{k}} \Psi_{c}\left(t_{k+1}, t_{k}+\tau\right)\left(x_{c}\left(t_{k}+\tau\right)-y_{c}\left(t_{k}+\tau\right)\right) d \tau\right\| \\
&+\left(\beta_{d c}^{f}+\beta_{d d}^{f}\right)\left\|x_{d}[k]-y_{d}[k]\right\| \\
&+2 r\left[\left(\beta_{c c}^{g}+\beta_{c d}^{g}\right)\left\|\int_{0}^{T_{k}} \Psi_{c}\left(t_{k+1}, t_{k}+\tau\right) d \tau\right\|+\beta_{d c}^{g}+\beta_{d d}^{g}\right] \quad \forall k \in \mathbb{Z}_{0+} \\
& \leq\left(\rho+\beta_{d c}^{f}+\beta_{d d}^{f}\right)\|x[k]-y[k]\| \\
&+r\left(\rho_{c} \bar{T}\left[\beta_{c c}^{f}+\beta_{c d}^{f}+2\left(\beta_{c c}^{g}+\beta_{c d}^{g}\right)\right]+2\left(\beta_{d c}^{g}+\beta_{d d}^{g}\right)\right) \quad \forall k \in \mathbb{Z}_{0+}
\end{aligned}
$$

since, by construction, $\left\|x_{d}[k]-y_{d}[k]\right\| \leq\|x[k]-y[k]\|$. Now, if

$$
\beta_{c c}^{f}=\beta_{c d}^{f}=\beta_{d c}^{f}=\beta_{d d}^{f}=\beta_{c c}^{g}=\beta_{c d}^{g}=\beta_{d c}^{g}=\beta_{d d}^{g}=0
$$

(i.e., all the perturbations are identically zero) and $\rho+\beta_{d c}^{f}+\beta_{d d}^{f}<1$, then from Schauder's first fixed point theorem on (5.14), the bounded sequence $\{x[k]\}_{k \in \mathbb{Z}_{0}^{+}}$has a unique fixed point in the convex closed $\bar{B}(0, r)$. One gets from (5.6) that

$$
\begin{aligned}
\|x(t)-y(t)\|_{\left[t_{k}, t_{k}+\tau\right]} \leq & \left(\left\|\bar{\Psi}_{c}\left(t_{k}+\tau, t_{k}\right)\right\|+\frac{K_{c}}{a_{c}}\left(1-e^{-a_{c} \tau}\right)\left(\beta_{c c}^{f}+\beta_{c d}^{f}\right)\right)\|x[k]-y[k]\| \\
& +2 \frac{K_{c}}{a_{c}}\left(1-e^{-a_{c} \tau}\right)\left(\beta_{c c}^{g}+\beta_{c d}^{g}\right) r \quad \forall \tau \in\left[0, T_{k}\right)
\end{aligned}
$$


for all $\tau \in\left[0, T_{k}\right)$, for all $k \in \mathbb{Z}_{0+}$. Thus, there is a unique fixed point $x^{*}(0)$ in $\bar{B}(0, r)$, then the state trajectory solution $\left(x_{c}^{T}(t), x_{d}^{T}[k]\right)^{T}: \mathbb{R}_{0+} \times \mathbb{R}^{n_{c}} \times \mathbb{Z}_{0+} \times \mathbb{R}^{n_{d}} \rightarrow \mathbb{R}^{n}$ has a fixed point $x^{*}(\tau)$ in $\bar{B}(0, r)$ for each $\tau \in[0, T)$. This point coincides with that of the real sequence $\{x[k]\}_{k \in \mathbb{Z}_{0}^{+}}$ for $\tau=0$ from (5.14). Property (iii) has been proven.

(iv) Assume that the constraints C4 are modified as follows:

$$
\begin{gathered}
g_{c c}\left(t, x_{c i}\right) \leq \beta_{c c}^{g} x_{c i} ; \quad g_{c d}\left(t, x_{d i}\right) \leq \beta_{c d}^{g} x_{d i}, \quad g_{d c}\left(t, x_{c i}\right) \leq \beta_{d c}^{g} x_{c i} ; \\
g_{d d}\left(t, x_{d i}\right) \leq \beta_{d d}^{g} x_{d i} ; \quad i=1,2 .
\end{gathered}
$$

Then, (5.14) and (5.16) are, respectively, modified as follows:

$$
\begin{aligned}
& \|x[k+1]-y[k+1]\| \\
& \quad \leq\left(\rho+\beta_{d c}^{f}+\beta_{d d}^{f}+\rho_{c} \bar{T}\left[\beta_{c c}^{f}+\beta_{c d}^{f}+\left(\beta_{c c}^{g}+\beta_{c d}^{g}\right)\right]+\beta_{d c}^{g}+\beta_{d d}^{g}\right)\|x[k]-y[k]\|, \\
& \|x(t)-y(t)\|_{\left[t_{k}, t_{k}+\tau\right]} \\
& \quad \leq\left(\left\|\bar{\Psi}_{c}\left(t_{k}+\tau, t_{k}\right)\right\|+\frac{K_{c}}{a_{c}}\left(1-e^{-a_{c} \tau}\right)\left(\beta_{c c}^{f}+\beta_{c d}^{f}+\beta_{c c}^{g}+\beta_{c d}^{g}\right)\right)\|x[k]-y[k]\|,
\end{aligned}
$$

for all $\tau \in\left[0, T_{k}\right)$, for all $k \in \mathbb{Z}_{0+}$ so that state trajectory solution $x: \mathbb{R}_{0+} \rightarrow \mathbb{R}^{n}$ possesses a unique fixed point $z^{*}(0) \in \bar{B}(0, r)$ for any harmless perturbations guaranteeing that

$$
\rho+\beta_{d c}^{f}+\beta_{d d}^{f}+\rho_{c} \bar{T}\left(\beta_{c c}^{f}+\beta_{c d}^{f}+\beta_{c c}^{g}+\beta_{c d}^{g}\right)+\beta_{d c}^{g}+\beta_{d d}^{g}<1
$$

provided that $\rho<1$. Also, assume that $\rho<1$ and the sequence of sampling periods $\left\{T_{k}\right\}_{k \in \mathbb{Z}_{0+}}$ converges asymptotically to a limit $T$ in $\left(\varepsilon_{T}, \bar{T}\right)$ for some sufficiently small upperbound $\bar{T}<1 / \rho_{c}\left(\beta_{c c}^{f}+\beta_{c d}^{f}+\beta_{c c}^{g}+\beta_{c d}^{g}\right)$, for all perturbations (5.17) constrained subject to any given additive constant $\left(\beta_{c c}^{f}+\beta_{c d}^{f}+\beta_{c c}^{g}+\beta_{c d}^{g}\right)$. Then, a unique fixed point $z^{*}(\tau) \in \bar{B}(0, r)$ exists for each $\tau \in[0, T)$ for any harmless perturbations subject to

$$
\rho+\beta_{d c}^{f}+\beta_{d d}^{f}+\beta_{d c}^{g}+\beta_{d d}^{g}<1-\rho_{c} \bar{T}\left(\beta_{c c}^{f}+\beta_{c d}^{f}+\beta_{c c}^{g}+\beta_{c d}^{g}\right)
$$

and Property (iv) has been proven.

Note that Theorem 5.1 is also applicable, in particular, for constant sampling periods.

Remark 5.2. Note that the fixed points of Theorem 5.1(iii)-(iv) are reached asymptotically for the state-trajectory solution for each $\tau \in[0, T)$ provided that the sequences of sampling instants and periods converge to finite limits. This does not mean that there is a unique equilibrium point for such a trajectory. The physical conclusion of Theorem 5.1(iii)-(iv) is that the unique asymptotically sable attractor might be either a globally asymptotically stable equilibrium point or a stable limit oscillation of period at most $T$. 
From Remark 5.2 and Theorem 5.1(iii)-(iv), one concludes the existence of globally stable equilibrium points or that of globally stable limit oscillations as follows in the next two particular results in view of (5.16) and (5.19).

Corollary 5.3. If $x^{*}(0)=0$ in Theorem 5.1(iii), then $x^{*}(\tau)=x^{*} \equiv 0$; for all $\tau \in[0, T)$. Then, $x(t) \rightarrow 0$ as $t \rightarrow \infty$ and zero is a globally asymptotically stable equilibrium point. If $z^{*}(0)=0$ in Theorem 5.1(iv) then, $x(t) \rightarrow z^{*} \equiv 0$, as $t \rightarrow \infty$ and zero is a globally asymptotically stable equilibrium point under the modified constraints $C 4^{\prime}$.

Corollary 5.4. Assume that $x^{*}\left(\tau_{1}\right) \neq x^{*}\left(\tau_{2}\right)$ for $\tau_{1}, \tau_{2}\left(\neq \tau_{1}\right) \in[0, T)$ in Theorem 5.1(iii). Then, any state-trajectory solution converges to a unique limit oscillation of period $T^{*} \leq T$ as $\mathbb{Z}_{0+} \ni k \rightarrow \infty$. If $x^{*}\left(\tau_{1}\right) \neq x^{*}\left(\tau_{2}\right)$ for $\tau_{1}, \tau_{2}\left(\neq \tau_{1}\right) \in[0, T)$ in Theorem 5.1(iv). Then, any state-trajectory solution converges to a unique limit oscillation $x(k T+\tau) \rightarrow z^{*}(\tau)$ as $\mathbb{Z}_{0+} \ni k \rightarrow \infty$ of period not larger that $T$; for all $\tau \in[0, T)$, under the modified constraints $C 4^{\prime}$.

The condition of convergence of the sequence of sampling periods to a constant limit is not necessary to derive Theorem 5.1(iii)-(iv) and Corollaries 5.3-5.4. The following ad-hoc generalization follows:

Corollary 5.5. Construct the continuous time argument $t:=t_{k}+\tau=\sum_{j=0}^{k-1} T_{j}+\tau$; for all $t \in\left[t_{k}, t_{k+1}\right)$; for all $\tau \in\left[0, T_{k}\right)$; for all $k \in \mathbb{Z}_{0+}$. Assume that $\lim _{k \rightarrow \infty}\left(\sum_{j=0}^{k} T_{j}+\tau_{1}(k)\right) \neq \lim _{k \rightarrow \infty}\left(\sum_{j=0}^{k} T_{j}+\right.$ $\left.\tau_{2}(k)\right)$ for $\tau_{1}(k), \tau_{2}(k)\left(\neq \tau_{1}(k)\right) \in\left[t_{k}, t_{k+1}\right)$ in Theorem 5.1(iii). Then, any state-trajectory solution converges to a unique limit oscillation of period $T^{*} \leq T$ as $\mathbb{Z}_{0+} \ni k \rightarrow \infty$.

Remark 5.6. An inequality for the maximum allowable $L_{\infty}$-norm of the error among any two state-trajectory solutions is now derived. Note from the constraints C.2-C.4, and (2.6)-(2.9) that

$$
\begin{aligned}
\left\|x_{d}[k+1]-y_{d}[k+1]\right\| & =\left\|A_{d}[k]\left(x_{d}[k]-y_{d}[k]\right)+A_{d c}[k]\left(x_{c}[k]-y_{c}[k]\right)+\delta_{d x}[k]-\delta_{d y}[k]\right\| \\
& \leq\left(m_{d}[k]+\beta_{d c}^{f}+\beta_{d d}^{f}\right)\|(\bar{x}[k]-\bar{y}[k])\|+2\left(\beta_{d c}^{g}+\beta_{d d}^{g}\right) r,
\end{aligned}
$$

where $\infty>\bar{m}_{d} \geq m_{d}[k]:=\left\|\left(A_{d c}[k], A_{d}[k]\right)\right\|$. Then, from (5.16) and (5.17), it is possible to take into account the discontinuities at sampling instants by evaluating the argument $\tau$ on the closed interval $\left[0, T_{k}\right]$ as follows:

$$
\begin{array}{r}
\|x(t)-y(t)\|_{\left[t_{k}, t_{k}+\tau\right]} \leq \operatorname{Max}_{0 \leq s \leq \tau}\left(\left\|\bar{\Psi}_{c}\left(t_{k}+s, t_{k}\right)\right\|+\frac{K_{c}}{a_{c}}\left(1-e^{-a_{c} \tau}\right)\left(\beta_{c c}^{f}+\beta_{c d}^{f}\right)+m_{d}[k]+\beta_{d c}^{f}+\beta_{d d}^{f}\right) \\
\times\|x(t)-y(t)\|_{\left[t_{k}, t_{k}+\tau\right]}+2\left(\frac{K_{c}}{a_{c}}\left(1-e^{-a_{c} \tau}\right)\left(\beta_{c c}^{g}+\beta_{c d}^{g}\right)+\beta_{d c}^{g}+\beta_{d d}^{g}\right) r \\
\forall \tau \in\left[0, T_{k}\right] .
\end{array}
$$


However, (5.23) leads to

$$
\begin{aligned}
\|x(t)-y(t)\| \leq & \|x-y\|_{[0, t]} \\
\leq & \left(\bar{\rho}_{c}+\frac{K_{c}}{a_{c}} \operatorname{Max}_{k \in \mathbb{Z}_{0+}} \operatorname{Max}_{\tau \in\left[0, T_{k}\right]}\left|1-e^{-a_{c} \tau}\right|\left(\beta_{c c}^{f}+\beta_{c d}^{f}\right)\right)\|x(t)-y(t)\|_{\infty} \\
& +\frac{2 K_{c}}{a_{c}}\left(\beta_{c c}^{g}+\beta_{c d}^{g}\right) r\left(\operatorname{Max}_{k \in \mathbb{Z}_{0+}} \operatorname{Max}_{\tau \in\left[0, T_{k}\right]}\left|1-e^{-a_{c} \tau}\right|\right) \\
\leq & \alpha\|x-y\|_{\infty}+\beta ; \quad \forall t \in \mathbb{R}_{0+},
\end{aligned}
$$

where

$$
\begin{gathered}
\alpha:=\bar{\rho}_{c}+\frac{K_{c}}{a_{c}} \underset{\tau \in[0, \bar{T}]}{\operatorname{Max}}\left|1-e^{-a_{c} \tau}\right|\left(\beta_{c c}^{f}+\beta_{c d}^{f}\right), \\
\beta:=\frac{2 K_{c}}{a_{c}}\left(\beta_{c c}^{g}+\beta_{c d}^{g}\right) r\left(\operatorname{Max}_{\tau \in[0, \bar{T}]}\left|1-e^{-a_{c} \tau}\right|\right), \quad \bar{\rho}_{c}:=\operatorname{Max}_{k \in \mathbb{Z}_{0+0} 0 \leq \tau \leq T_{k}}\left\|\bar{\Psi}_{c}\left(t_{k}+\tau, t_{k}\right)\right\| .
\end{gathered}
$$

Then, $\|x-y\|_{\infty} \leq \operatorname{Max}\left(2 r,\left(1-\alpha^{-1}\right) \beta\right)$ if $\alpha<1$.

\section{Acknowledgments}

The author is very grateful to the Spanish Ministry of Education by its partial support of this work through Project DPI2006-00714. He is also grateful to the Basque Government by its support through Grants GIC07143-IT-269-07 and SAIOTEK S-PE08UN15.

\section{References}

[1] W. S. Kafri and E. H. Abed, "Stability analysis of discrete-time singularly perturbed systems," IEEE Transactions on Circuits and Systems, vol. 43, no. 10, pp. 848-850, 1996.

[2] L. V. Kolev, Interval Methods for Circuit Analysis, vol. 1 of Advanced Series on Circuits and Systems, World Scientific, River Edge, NJ, USA, 1993.

[3] L. S. Shieh, J. S. H. Tsai, and S. R. Lian, "Determining continuous-time state equations from discretetime state equations via the principal qth root method," IEEE Transactions on Automatic Control, vol. 31, no. 5, pp. 454-457, 1986.

[4] E. P. Oppenheimer and A. N. Michel, "Application of interval analysis techniques to linear systems. II. The interval matrix exponential function," IEEE Transactions on Circuits and Systems, vol. 35, no. 10, pp. 1230-1242, 1988.

[5] E. P. Oppenheimer and A. N. Michel, "Application of interval analysis techniques to linear systems. III. Initial value problems," IEEE transactions on circuits and systems, vol. 35, no. 10, pp. 1243-1256, 1988.

[6] M. De la Sen, "The reachability and observability of hybrid multirate sampling linear systems," Computers \& Mathematics with Applications, vol. 31, no. 1, pp. 109-122, 1996.

[7] P. T. Kabamba and S. Hara, "Worst-case analysis and design of sampled-data control systems," IEEE Transactions on Automatic Control, vol. 38, no. 9, pp. 1337-1357, 1993.

[8] M. De la Sen and C. F. Alastruey, "Stability results for two classes of linear time-delay and hybrid systems," Engineering Computations, vol. 21, no. 7, pp. 718-735, 2004.

[9] M. De la Sen, "Sufficiency-type stability and stabilization criteria for linear time-invariant systems with constant point delays," Acta Applicandae Mathematicae, vol. 83, no. 3, pp. 235-256, 2004. 
[10] M. De la Sen and N. Luo, "On the uniform exponential stability of a wide class of linear time-delay systems," Journal of Mathematical Analysis and Applications, vol. 289, no. 2, pp. 456-476, 2004.

[11] C. Chen and C. Zhu, "Fixed point theorems for $n$ times reasonable expansive mapping," Fixed Point Theory and Applications, vol. 2008, Article ID 302617, 6 pages, 2008.

[12] L.-G. Hu, "Strong convergence of a modified Halpern's iteration for nonexpansive mappings," Fixed Point Theory and Applications, vol. 2008, Article ID 649162, 9 pages, 2008.

[13] S. Saeidi, "Approximating common fixed points of Lipschitzian semigroup in smooth Banach spaces," Fixed Point Theory and Applications, vol. 2008, Article ID 363257, 17 pages, 2008.

[14] M. De la Sen, "About robust stability of dynamic systems with time delays through fixed point theory," Fixed Point Theory and Applications, vol. 2008, Article ID 480187, 20 pages, 2008.

[15] A. Latif and A. A. N. Abdou, "Fixed points of generalized contractive maps," Fixed Point Theory and Applications, vol. 2009, Article ID 487161, 9 pages, 2009.

[16] J.-Z. Xiao and X.-H. Zhu, "Common fixed point theorems on weakly contractive and nonexpansive mappings," Fixed Point Theory and Applications, vol. 2008, Article ID 469357, 8 pages, 2008.

[17] D. Kincaid and W. Cheney, Numerical Analysis, Mathematics of Scientific Computing, Brooks/Cole, Belmont, Calif, USA, 1991.

[18] J. K. Hale, Ordinary Differential Equations, vol. 20 of Pure and Applied Mathematics, Wiley-Interscience, New York, NY, USA, 1969. 